\title{
Mitigation of modulation instability in Brillouin distributed fiber sensors by using orthogonal polarization pulses
}

\author{
Javier Urricelqui ${ }^{\mathrm{a}}$, Mehdi Alem ${ }^{\mathrm{b}}$, Mikel Sagues ${ }^{\mathrm{a}}$, Luc Thévenaz ${ }^{\mathrm{b}}$, Alayn Loayssa ${ }^{\mathrm{a}}$, Marcelo A. Soto ${ }^{\mathrm{b}^{*}}$ \\ aUniversidad Pública de Navarra, Campus Arrosadia s/n, 31006 Pamplona, Spain; \\ ${ }^{\mathrm{b}}$ EPFL Swiss Federal Institute of Technology, Institute of Electrical Engineering \\ SCI STI LT, Station 11, CH-1015 Lausanne, Switzerland \\ *E-mail: marcelo.soto@epfl.ch
}

\begin{abstract}
A technique based on the use of orthogonally-polarized pulses is proposed to mitigate the detrimental impact of modulation instability on Brillouin distributed fiber sensors. While the theoretical underpinnings of the method are described by introducing a detailed model for the vector modulation instability, the technique is experimentally validated in a $25-\mathrm{km}$ sensing link. Numerical and experimental results demonstrate that the use of orthogonally-polarized pulses not only mitigates the impact of modulation instability, but also the four-wave mixing occurring in systems using pumps with parallel polarization; thus, providing an important sensing range enhancement with a reduced pump depletion.
\end{abstract}

Keywords: Distributed fiber sensor, stimulated Brillouin scattering, modulation instability, Manakov system

\section{INTRODUCTION}

The sensing range achieved by Brillouin optical time domain analyzers (BOTDA) has been remarkably increased during the last decade ${ }^{1}$. Optimizing the system to reach very long sensing ranges actually requires launching high pump and probe powers into the sensing fiber to provide a sufficient signal-to-noise ratio (SNR) on the measurements at the far end of the fiber. However, increasing the input power above a critical level excites undesired nonlinear effects such as modulation instability (MI) and stimulated Raman scattering (SRS), which deplete the pump and reduce the maximum sensing range of the system ${ }^{2,3}$. Compared with SRS, MI shows a lower threshold and thus imposes a more restrictive limitation to the maximum pump power in BOTDA schemes ${ }^{2,3}$. Recently, techniques based on the use of multi-wavelength pulses have been proposed to extend the measurement range ${ }^{4}$, to provide high spatial resolution ${ }^{5}$ or to enable fast dynamic sensing . $^{6}$ However, it has been identified in all these cases that nonlinear cross Kerr-effect interactions between the different spectral lines would take place ${ }^{4,6}$ if high-power pulses are simultaneously launched into the sensing fiber, leading to severe distortions of the measured time-domain traces. An intuitive partial solution to avoid the reported Kerr interactions ${ }^{4}$ is to launch into the fiber two orthogonally-polarized pulses7. This would, in principle, allow increasing the total pump power without inducing Kerr nonlinearities, thus leading to an improvement in the sensor response.

This paper presents a thorough theoretical and experimental analysis of the Kerr interactions between two high-power pump pulses in a BOTDA system. Results demonstrate that the use of orthogonal pumps in a BOTDA sensor highly mitigates the impact of Kerr nonlinearities in comparison to the use of a single pump or parallel pumps; however, the total power of orthogonally-polarized pulses is still limited by nonlinear cross-interactions that obey a Manakov model ${ }^{8,9}$. Experimental results, supported by numerical simulations, demonstrate an increment of the MI threshold, which leads to a reduction of the depletion level. Although the study is here carried out using a specific BOTDA scheme based on the RF detection of a phase-modulated probe ${ }^{7}$, the analysis and method reported in this paper can be perfectly applied to other Brillouin distributed fiber sensor schemes, where the response of the signal is directly scaled by the pump power evolution.

\section{MANAKOV MODEL FOR ORTHOGONALLY-POLARIZED PULSES}

The limitations imposed by modulation instability in standard BOTDA systems have been investigated thoroughly based on the scalar nonlinear Schrödinger equation (NLSE) ${ }^{3}$. To take into account the interaction between orthogonally-polarized pumps, the scalar analysis of MI needs to be extended to its vector counterpart, where the vector NLSE (VNLSE) governs the polarization components of the lightwave. The form of the VNLSE depends on the birefringence characteristics of the fiber. Conventional single-mode fibers (SMFs) are categorized as 'randomly varying birefringent fibers', meaning that 
their birefringence changes randomly over a length scale of $\sim 10 \mathrm{~m}$ in both value and axes ${ }^{8}$. The random fluctuations in the degree of modal birefringence and the orientation of birefringence axes force the Stokes vector to sweep the entire surface of the Poincaré sphere over a 1 km length scale. For optical fibers longer than this length scale, averaging the VNLSE over the Poincaré sphere leads to the following coupled nonlinear Schrödinger equations referred to as the Manakov system for the two orthogonal polarization components $u(z, t)$ and $v(z, t)^{8,9}$ :

$$
\left\{\begin{array}{l}
\frac{\partial u}{\partial z}+i \frac{\beta_{2}}{2} \frac{\partial^{2} u}{\partial t^{2}}+\frac{\alpha}{2} u=i \gamma\left(|u|^{2}+|v|^{2}\right) u \\
\frac{\partial v}{\partial z}+i \frac{\beta_{2}}{2} \frac{\partial^{2} v}{\partial t^{2}}+\frac{\alpha}{2} v=i \gamma\left(|u|^{2}+|v|^{2}\right) v
\end{array},\right.
$$

where $\alpha$ is the fiber attenuation and $\beta_{2}<0$ is the group-velocity dispersion (GVD) coefficient. Note that to take into account the randomness of polarization ${ }^{8}$, the nonlinear coefficient $\gamma$ is in this case obtained multiplying the linearlypolarized Kerr nonlinearity by the factor 8/9. It is evident from the right-hand side of Eq. (1) that both polarizations contribute equally to the nonlinear phase modulation of the optical waves. Therefore, under the same total power conditions, the MI spectral bands originated by a single pump and a double orthogonal pump scheme are the same. However, since in the latter case the total power is split into two polarizations at different wavelengths, the peak power and thus the MI gain turn out to be reduced.

To verify the above-described situation, a numerical analysis of Eq. (1) has been carried out using the split-step Fourier method for solving the VNLSE and a Monte Carlo simulation for considering the background noise as a seeding signal for the onset of MI. Fig. 1(a) depicts the MI gain spectrum originated in a $25 \mathrm{~km}$-long standard SMF with a total input pump power of $250 \mathrm{~mW}$, under three different scenarios: the standard single pulse case at zero-frequency detuning (blue curve) is compared with the use of two pulses at $\pm 10 \mathrm{GHz}$ having parallel (green curve) and orthogonal (red curve) states of polarization. It should be noticed that the pumps lines are removed from the plots for the sake of visibility. It is possible to observe that while the MI gain in the single pump case is higher than the one of the orthogonal polarizations, an efficient four-wave mixing (FWM) process dominates the interaction between the pulses with parallel polarizations. Fig. 1(b) shows the evolution of the total pump power along the fiber for the three scenarios (note that the fiber attenuation has been compensated for a better visualization). While the single pump power is substantially depleted by MI (about $70-80 \%$ of depletion), the case of parallel pumps suffers from strong FWM, leading to abrupt pump power oscillations along the fiber. On the other hand, it is interesting to observe the behavior of the double orthogonal pumps, which shows a considerable improvement in terms of MI pump depletion compared to the standard single pump configuration, but also offers a significant suppression of FWM when compared to the parallel pumps.
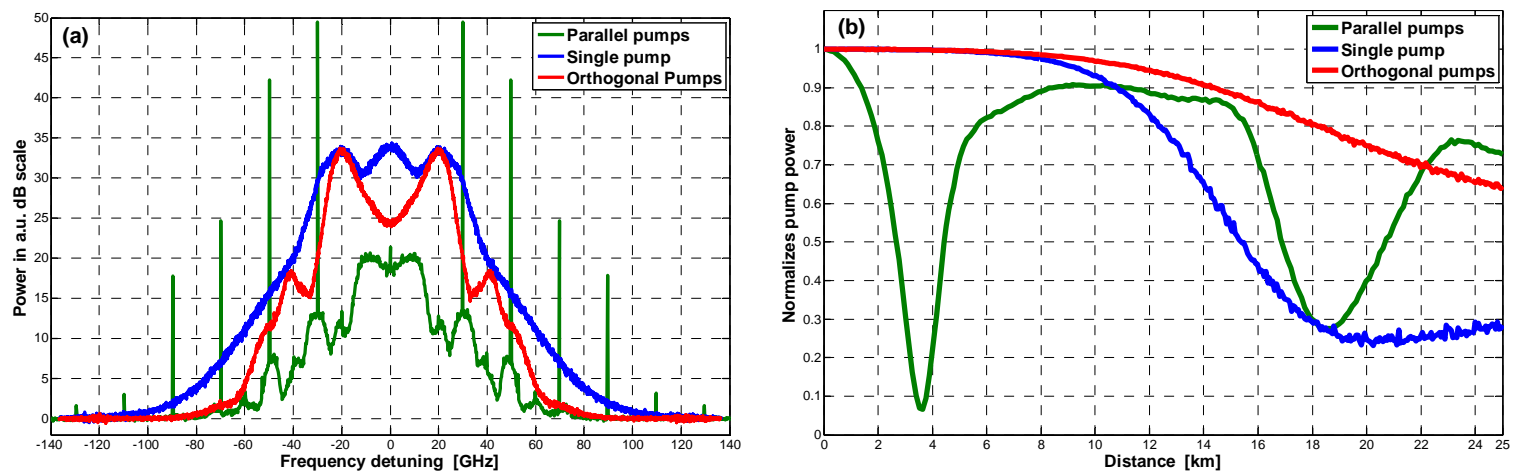

Figure 1. (a) MI gain spectrum and (b) longitudinal pump power evolution along the fiber, for a $25 \mathrm{~km}$-long SMF with $\alpha=$ $0.2 \mathrm{~dB} / \mathrm{km}, \gamma=1.8 \mathrm{~W}^{-1} / \mathrm{km}$ and $\beta_{2}=-22 \mathrm{ps}^{2} / \mathrm{km}$, in three different pump scenarios: single-wavelength pump (blue curves), two parallel pumps (green curves), and two orthogonal pumps (red curves). The frequency spacing between pumps is $20 \mathrm{GHz}$, the noise power spectral density is $-118 \mathrm{dBm} / \mathrm{Hz}$, and total power is $250 \mathrm{~mW}$.

\section{EXPERIMENTAL SETUP AND RESULTS}

Fig. 2(a) depicts the experimental setup assembled to demonstrate the reduction of Kerr nonlinearities by using orthogonal pump pulses in BOTDA sensors. The output of a laser source is divided in two optical branches with an optical coupler. In the upper branch, optical pump pulses are shaped using a Mach-Zehnder electro-optic modulator (MZ-EOM) driven by a $9.55 \mathrm{GHz}$ pulsed microwave signal and biased in minimum transmission. This way, two pulsed optical sidebands are 
generated, while the optical carrier is highly suppressed. This optical signal goes through a polarization controller (PC), a differential group delay (DGD) module and a programmable optical filter (POF) shown inside a dashed box in Fig. 2(a). These optical components actually allow setting the three-above mentioned scenarios for the study of MI, i.e. orthogonal pump pulses, parallel pulses and single pulse. The polarization controller allows the linearly-polarized light at the output of the MZ-EOM to be properly aligned into the DGD module. This birefringent material generates a wavelength-dependent phase shift difference between its axes given by ${ }^{7}: \Delta \theta=2 \pi \Delta f \cdot \Delta \tau$, where $\Delta \tau$ is the differential group delay introduced by birefringence in the DGD module and $\Delta f$ is the frequency spacing between the optical waves. Therefore, depending on the wavelength difference and the incident angle, the birefringent material can provide different polarization states at its output. On the one hand, two orthogonally-polarized pulses can be obtained if the incident angle into the birefringent material is set to $45^{\circ}$ and $\Delta \theta=\pi$. In our setup, this last condition is easily fulfilled by choosing a DGD module with $\Delta \tau=26$ ps. On the other hand, the generation of pulses with parallel polarizations only requires to adjust the incident angle to one of the principal axes of the DGD module. Finally, for a standard single-wavelength pulse, the programmable optical filter is adjusted to select only one of the sidebands. Once the desired pump signal is generated, this is amplified by an erbium doped fiber amplifier (EDFA), whose amplified spontaneous emission noise is filtered out by a 1-nm optical filter.
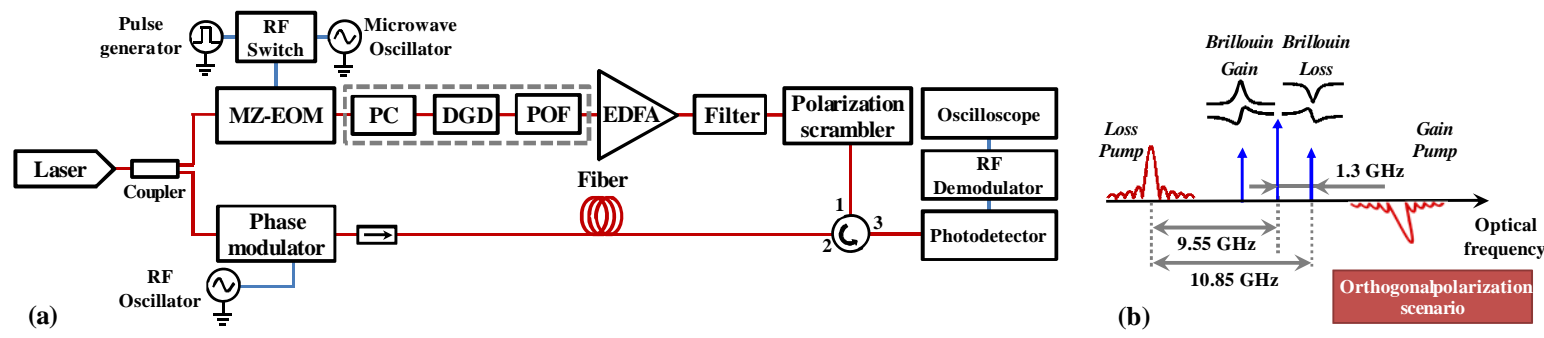

Figure 2. (a) Experimental BOTDA sensor and (b) Brillouin interactions for the orthogonal pump pulses case.

In the lower branch, the probe wave is generated with an electro-optic phase modulator driven by a $1.3 \mathrm{GHz} \mathrm{RF}$ signal. This modulation frequency is chosen so that the upper (lower) sideband of the phase modulation interacts with the lower (higher) pulsed sideband, so that a cross-interaction between the different sidebands occurs as shown in Fig. 2(b). After interacting via stimulated Brillouin scattering, the probe signal is directed to a photodetector, where the resulting RF signal is then demodulated ${ }^{7}$. Finally, the BOTDA signal is captured in a digital oscilloscope.

Fig. 3(a) shows the measured (continuous lines) and simulated (dashed lines) time-domain traces obtained in a BOTDA sensor using a single pulse and orthogonally-polarized pulses for a total input power of $300 \mathrm{~mW}$. Clear differences in the pump power evolution can be observed, especially in the last $10 \mathrm{~km}$ of fiber, where the power of the single pump is depleted by $\sim 50 \%$ as a result of scalar MI, while the total power in the two orthogonal pulses is only depleted by $12 \%$ due to vector MI. This represents a $\sim 3 \mathrm{~dB}$ improvement in the sensor response at the end of the fiber, enabling a sensing range extension of $\sim 7.5 \mathrm{~km}$ while maintaining the same SNR ${ }^{1}$. An excellent agreement between the experimental and numerical results is observed, confirming the validity of the model presented in Eq. (1). As a comparison, Fig. 3(b) shows the trace evolution obtained using pulses with parallel polarization and exactly the same power as in Fig. 3(a). The trace is clearly affected
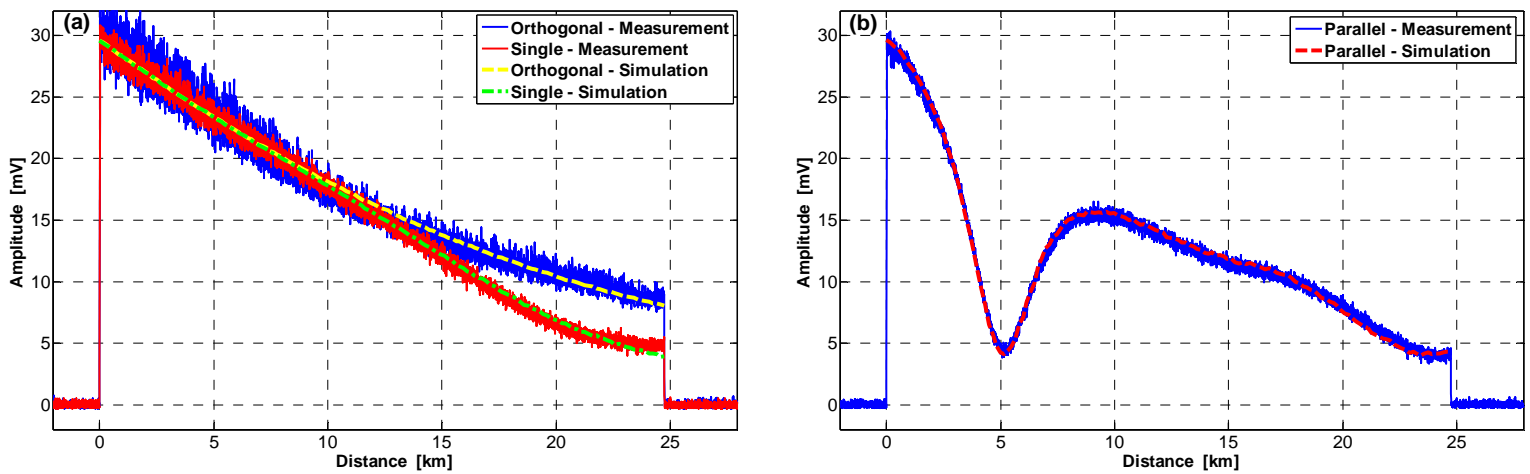

Figure 3. Measured and simulated time-domain Brillouin traces in a 25-km SMF for an input power of $300 \mathrm{~mW}$ for three cases: (a) single pump and double orthogonal pumps, and (b) parallel pulses. The parameters used to solve Eq. (1) are: $\alpha=0.2 \mathrm{~dB} / \mathrm{km}, \gamma=1.0 \mathrm{~W}^{-1} / \mathrm{km}$ and $\beta_{2}=-23 \mathrm{ps}^{2} / \mathrm{km}$. The frequency difference between pumps is $19.1 \mathrm{GHz}$ and the noise power spectral density is $-109 \mathrm{dBm} / \mathrm{Hz}$. 
by FWM, which induces oscillations of the pump power along the fiber. This situation highlights another advantage of using orthogonal pulses, since they can efficiently mitigate the impact of FWM, as predicted by the Manakov model ${ }^{9}$. In addition, small differences in the polarization fading should be observed between the traces depicted in Fig. 3; however this is only caused by the polarization diversity method used in each scheme. Thus, while a polarization scrambler is required in the cases of single and parallel pulses, the use of orthogonal pulses does not require additional polarization scrambling since it ensures two complementary Brillouin interactions taking place at each position of the sensing fiber

In order to evaluate the performance improvement provided by the use of orthogonal pulses, the measured depletion ratio at the end of the fiber is compared with the single pulse case for different input pump powers. As shown in Fig. 4, the depletion ratio using a single pulse is clearly much higher than when using orthogonally-polarized pulses. For instance, tolerating a depletion ratio of $\sim 15 \%$, the input pump power can be increased from about $220 \mathrm{~mW}$ to $320 \mathrm{~mW}$ using pulses with orthogonal polarizations, representing a 1.5-fold improvement in the input pump power supported by the sensor before inducing distortions in the time-domain traces. Under a pump power of $320 \mathrm{~mW}$, the depletion ratio grows from $15 \%$ using orthogonal polarization up to $53 \%$ using the single pulse, which also reaffirms the advantage of using pulses with orthogonal polarizations.

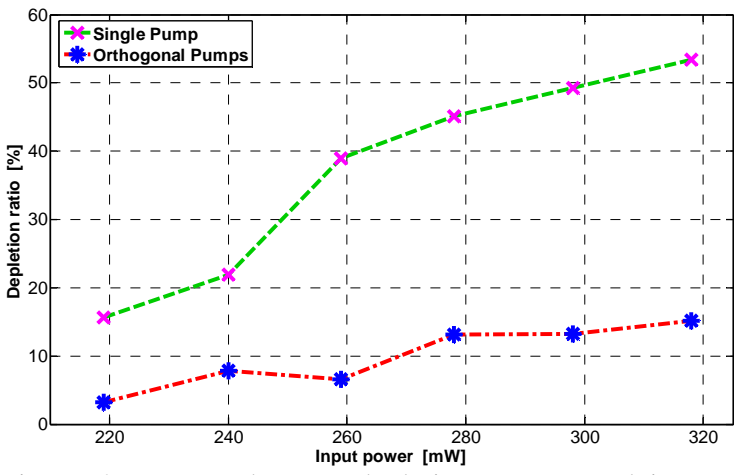

Figure 4. Measured pump depletion versus total input power at $25 \mathrm{~km}$ distance, for two cases: single pump (crosses), and double orthogonal pumps (stars).

\section{CONCLUSIONS}

A compensation of the MI effect on BOTDA systems has been experimentally and theoretically demonstrated by using pulses with orthogonal states of polarization. Results indicate that Kerr nonlinearities between orthogonally-polarized pulses are not completely null, and the maximum pump power allowed by a BOTDA system is in this case determined by the Manakov model for the Kerr effect. The presented study is useful to understand the power limitations in many Brillouin distributed sensors, also offering the possibility to properly design a system with higher pump power than the well-known threshold imposed by modulation instability in conventional single-wavelength pump schemes, at least for distance ranges over which the orthogonality of polarized waves separated by several GHz in low birefringence fibers can be maintained.

J. Urricelqui, M. Sagues and A. Loayssa acknowledge the support from the Spanish Ministerio de Economia y Competitividad through the project TEC2013-47264-C2-2-R, FEDER funds and Universidad Pública de Navarra. M. Alem, L. Thévenaz and M. A. Soto acknowledge the support from the Swiss Commission for Technology and Innovation (Project 13122.1).

\section{REFERENCES}

[1] Soto, M. A. and Thévenaz, L., "Modeling and evaluating the performance of Brillouin distributed optical fiber sensors," Opt. Express 21(25), 31347-31366 (2013).

[2] Foaleng, S. M. and Thévenaz, L., "Impact of Raman scattering and modulation instability on the performances of Brillouin sensors," Proc. of SPIE 7753, 77539V (2011).

[3] Alem, M., Soto, M. A. and Thévenaz, L., "Modelling the depletion length induced by modulation instability in distributed optical fibre sensors,” Proc. of SPIE 9157, 91575S (2014).

[4] Soto, M. A., et al., "Time and frequency pump-probe multiplexing to enhance the signal response of Brillouin optical time-domain analyzers,” Opt. Express, 22(23), 2858428595 (2014).

[5] Motil, A., Danon, O., Peled, Y. and Tur, M., "High spatial resolution BOTDA using simultaneously launched gain and loss pump pulses," Proc. of SPIE 8794, 87943L (2013).

[6] Voskoboinik, A., Yilmaz, O., Willner, A. and Tur, M., "Sweep-free distributed Brillouin time-domain analyzer (SF-BOTDA),” Opt. Express 19(26), B842-B847 (2011).

[7] Urricelqui, J., López-Fernandino, F., Sagues, M. and Loayssa, A., "Polarization diversity scheme for BOTDA sensors based on a double orthogonal pump interaction,” To be published in J. Lightwave Technol. (2015).

[8] Wai, P. K. A. and Menyuk, C. R., "Polarization mode dispersion, decorrelation, and diffusion in optical fibers with randomly varying birefringence,” J. Lightwave Technol. 14(2), 148-157 (1996).

[9] Kibler, B., et al., "Observation of Manakov polarization modulation instability in the normal dispersion regime of randomly birefringent telecom optical fiber," Proc. of IEEE, pp.1-3, ECOC, Cannes, France (2014). 